\title{
Guía de Práctica Clínica para el Diagnóstico y Tratamiento de Esclerosis Múltiple en Adultos.
}

\author{
Clinical Practice Guidelines: Diagnostic and Treatment of Multiple Sclerosis in Adults
}

\begin{abstract}
Darwin R. Vizcarra ${ }^{1,2, a, b}$, Ana G. Cruz ${ }^{3,4, a}$, Edgar Rojas ${ }^{5,6, a}$, Nicanor Mori ${ }^{7, a}$, César Caparón ${ }^{8, a}$, Carlos C. Castañeda $^{9, a}$, Sheila Castro ${ }^{10, a}$, Jorge M. Gavidiaa ${ }^{11,12, a}$, Oscar González ${ }^{6,13, a}$, Marco Huertas ${ }^{5,14, a}$, María Meza Víctor Montalván ${ }^{5, \mathrm{a}}$, Alda Rivara ${ }^{6,15, \mathrm{a}}$, Fiorella Adrianzén ${ }^{1,2, c}$, Kevin Duque $^{1,2, c}$, Sebastián A. Serván ${ }^{1,2, c}$, Brian J. Villafuerte 1 1,2, , Joaquín A. Vizcarraa ${ }^{1,2, c}$, Rodrigo Zamudio ${ }^{1,2, c}$, Elsa R. Neira ${ }^{2,9, d}$, Adriana Carrá16,a, Oscar Fernández $^{17,18, e}$, Víctor Rivera ${ }^{19, a}$
\end{abstract}

\section{RESUMEN}

La Esclerosis Múltiple (EM) es una enfermedad crónica del sistema nervioso central, para la cual aún no hay una cura definitiva; sin embargo, existe una diversa variedad de terapias con el objetivo de modificar el curso natural de la enfermedad, que promueve la inclusión constante de nuevas estrategias terapéuticas. Objetivo: La Sociedad Peruana de Neurología, por encargo del Ministerio de Salud, convocó a un comité de expertos con el objetivo de elaborar una guía de práctica clínica para el diagnóstico y tratamiento de EM. Método: Se realizó una búsqueda y evaluación de guías de práctica clínica bajo la metodología AGREE II, escogiendo como modelo la Guía de Práctica Clínica Catalana. Las preguntas clínicas no concernientes al tratamiento fueron resueltas a través de revisión sistemática. Las preguntas clínicas de tratamiento se diseñaron bajo el formato PICO y se resolvieron con un meta-análisis de ensayos clínicos disponibles hasta agosto del 2017, tomando en consideración las terapias aprobadas por DIGEMID hasta enero del 2017. Las recomendaciones finales fueron elaboradas mediante el método Delphi modificado con un consenso de al menos $80 \%$ de los miembros de su comité. Finalmente se realizó una revisión externa del manuscrito

\footnotetext{
Hypnos Instituto del Sueño-Clínica San Felipe. Lima, Perú.

Universidad Peruana Cayetano Heredia. Lima, Perú.

Hospital Nacional Alberto Sabogal Sologuren. Callao, Perú.

Universidad de San Martín de Porres. Lima, Perú

Hospital Nacional Guillermo Almenara Irigoyen. Lima, Perú.

Universidad Nacional Mayor de San Marcos. Lima, Perú.

Hospital Nacional Daniel Alcides Carrión. Lima, Perú.

Hospital Nacional LNS Policía Nacional del Perú. Lima, Perú.

9 Hospital Nacional Cayetano Heredia. Lima, Perú.

10 Instituto Nacional de Ciencias Neurológicas. Lima, Perú.

11 Clínica Angloamericana. Lima, Perú.

12 Universidad Peruana de Ciencias Aplicadas. Lima, Perú.

13 Policlínico Peruano Japonés. Lima, Perú.

14 Universidad San Juan Bautista. Lima, Perú

15 Hospital Nacional Edgardo Rebagliati Martins.

16 Hospital Británico, Neurociencias Fundación Favaloro INECO. Buenos Aires, Argentina

${ }_{17}$ Facultad de Medicina de Málaga. Malaga, España,

18 Instituto de Investigación Biomédica de Málaga (IBIMA) Hospital Regional Universitario de Málaga. Malaga, España.

19 Departamento de Neurología Baylor College of Medicine. Houston,Texas, USA.

a Neurólogo Clínico, ${ }^{b}$ Especialista en Medicina del Sueño, ${ }^{c}$ Médico Cirujano, ${ }^{d}$ Endocrinóloga

e Investigador Senior
} 
Guía de Práctica Clínica para el Diagnóstico y Tratamiento de Esclerosis Múltiple en Adultos.

por expertos internacionales en EM. Resultados: Se formularon 18 preguntas clínicas y 21 recomendaciones para el manejo, incluyendo algoritmos terapéuticos.

PALABRAS CLAVE: Esclerosis múltiple, guía de práctica clínica, Perú.

\section{SUMMARY}

Multiple Sclerosis (MS) is a chronic disease of the central nervous system, for which there is still no definitive cure; but there is a diverse variety of therapies with the objective of modifying the course of the disease, which promotes the constant inclusion of new therapeutic strategies. Objective: The Peruvian Society of Neurology, as requested by the Peruvian Health Ministry, convened a committee of experts with the purpose of elaborating a clinical practice guideline for the diagnosis and treatment of MS. Method: Clinical practice guidelines were searched and evaluated according to the AGREE II methodology, choosing the Catalan Clinical Practice Guide as a model. The clinical questions not related to treatment were solved through a systematic review. The clinical treatment questions were assessed under the PICO format and were solved with a meta-analysis of clinical trials available until August 2017, considering the therapies approved by DIGEMID until January 2017. The final recommendations were elaborated using the modified Delphi method with a consensus of at least $80 \%$ of the members of its committee. Finally, an external revision of the manuscript was made by international experts in MS. Results: Eighteen clinical questions and twenty-one recommendations for management were developed, including therapeutic algorithms.

KEYWORDS: Multiple sclerosis, practice guideline, Peru.

\section{INTRODUCCIÓN}

La esclerosis múltiple (EM) es una enfermedad crónica inflamatoria y desmielinizante del sistema nervioso central. No se tiene una cura definitiva hasta el momento, por lo que el tratamiento está dirigido hacia modificar el curso natural de la enfermedad. En la última década se ha producido un rápido desarrollo en la investigación de nuevas terapias modificadoras de la enfermedad (TME) para pacientes con EM. Actualmente, se dispone de al menos catorce TME en el mercado internacional, once de ellas han sido aprobadas para su uso en el Perú. La celeridad con que son introducidas en la práctica clínica exige un constante esfuerzo por renovar la estrategia terapéutica. Un amplio arsenal de nuevas drogas hace de esta elección una decisión tan compleja como diversa. Esta diversidad se ve reflejada en los varios manejos terapéuticos propuestos para pacientes con EM.

El objetivo de este trabajo fue elaborar recomendaciones que sirvan de guía para profesionales de la salud que atienden pacientes adultos con EM. Estas fueron propuestas en consenso por un grupo de expertos con la finalidad de reducir la morbilidad asociada a esta enfermedad. Las recomendaciones fueron diseñadas sobre la base de la evidencia científica que evaluó la eficacia, seguridad y tolerabilidad de todas las TME aprobadas por la autoridad de Salud hasta enero del 2017. Se propusieron algoritmos para el tratamiento.

Este artículo es un resumen de un documento más amplio titulado "Guía Técnica: Guía de Práctica Clínica para el Diagnóstico y Tratamiento de Esclerosis Múltiple en Adultos", y tiene como objetivo su difusión. La versión completa está disponible a través de la página web de la Sociedad Peruana de Neurología (SPN) en http://www.spneurologia.org. pe/?q=node $/ 142$.

\section{Metodología y Estrategia de Búsqueda}

Por oficio $\mathrm{N}^{\circ} 2093$ emitido el 2015 por el Ministerio de Salud del Perú, se a la SPN la elaboración de la Guía de Práctica Clínica para el Diagnóstico y Tratamiento de EM. El grupo de trabajo estuvo constituido por un comité directivo, comité clínico, comité investigador y panel de revisores externos. El liderazgo y organización del proyecto recayó sobre el comité directivo, organizado por la SPN. El comité clínico estuvo conformado por miembros de la SPN, para lo cual se hizo una invitación abierta a todos los miembros que desearan participar, y que en virtud a su experiencia en el manejo de pacientes con EM fueran considerados expertos bajo la siguiente definición: aquel cuya formación y experiencia previa le ha 
permitido alcanzar un dominio sobre un asunto que excede el nivel promedio de sus iguales.

El desarrollo de la guía estuvo dirigido hacia responder las preguntas clínicas planteadas por este grupo de expertos, bajo cumplimiento de las directivas de la norma técnica para elaboración de guías de práctica clínica del Ministerio de Salud (1). De acuerdo con estas directivas, se decidió hacer una adaptación de guías existentes. Se realizó una búsqueda inicial de todas las guías disponibles en organismos y bases de datos: NGC (National Guideline Clearinghouse, Centro Nacional de Guías de EEUU), NHS MeLM (National Electronic Library for Medicines), Guiassalud de España, NICE (National Institute for Health and Clinical Excellence), SIGN (Scottish Intercollegiate Guidelines Network), UpToDate, EMA (Agencia Europea de Medicamento), FDA (The Food and Drug Administration), Pubmed/MEDLINE, EMBASE, Scopus, The Cochrane Library, Scielo
(Scientific Electronic Library Online), LILACS (Literatura Latinoamericana y del Caribe en Ciencias de la Salud), HINARI, BIREME y LIPECS (Literatura Peruana en Ciencias de la Salud). La evaluación de estas guías se hizo bajo la metodología AGREE II (Appraisal of Guidelines for Research \& Evaluation II) por miembros del comité directivo y clínico (2). Dicha evaluación, encontró a la Guía de Práctica Clínica Catalana como la mejor calificada, la cual se tomó como modelo (3). Las preguntas clínicas que abordaron aspectos como la epidemiología, factores de riesgo, consideraciones previas al inicio del tratamiento, criterios diagnósticos y exámenes auxiliares, fueron respondidas por el comité investigador mediante la revisión sistemática de la evidencia (tabla 1).

Adicionalmente, se generaron preguntas clínicas diseñadas bajo el formato PICO (Poblation, Intervention, Comparator, Outcome) en aquellas

Tabla 1. Preguntas clínicas.

¿Cuáles son los aspectos epidemiológicos de la EM y sus factores de riesgo?

¿Cuál es el cuadro clínico de la EM?

En pacientes con síndrome clínico aislado, ¿es posible identificar factores de riesgo para progresión hacia esclerosis múltiple clínicamente definida?

En pacientes con esclerosis múltiple clínicamente definida, ¿es posible identificar factores de riesgo para progresión de la enfermedad o para enfermedad agresiva?

¿Cómo se realiza el diagnóstico de EM?

¿Cuál es el rol de los exámenes auxiliares para el diagnóstico de EM?

¿Qué consideraciones se deben tener en cuenta previo al inicio de terapia modificadora de la enfermedad?

¿Cuáles es el concepto de EM de inicio agresivo y su importancia?

¿Cuál es la conducta terapéutica a seguir en mujeres con deseo gestacional, gestantes y puérperas con esclerosis múltiple?

En los pacientes con EM en terapia modificadora de la enfermedad, ¿cuál es el riesgo de desarrollar enfermedad tuberculosa primaria o reactivada?

¿Cuáles son los criterios de respuesta subóptima y la importancia de la adherencia a la terapia modificadora de la enfermedad?

¿Cuáles son las condiciones de escalamiento o cambio lateral en la terapia modificadora de la enfermedad en pacientes con EM?

¿Cuál es el marco de la prescripción de los medicamentos biosimilares?

¿Existe riesgo de exacerbaciones en pacientes con esclerosis múltiple luego de la vacunación?

¿Cuáles son los tratamientos disponibles para el brote de la enfermedad y cuál es el más adecuado?

En pacientes con síndrome clínico aislado ¿cuál es el perfil de eficacia y seguridad de las terapias modificadoras de la enfermedad?

¿Cuál es el perfil de eficacia y seguridad de las terapias modificadoras de la enfermedad para el tratamiento de la esclerosis múltiple remitente-recurrente?

¿Cuál es el perfil de eficacia y seguridad de las terapias modificadoras de la enfermedad para el tratamiento de la esclerosis múltiple primariamente y secundariamente progresiva? 
Guía de Práctica Clínica para el Diagnóstico y Tratamiento de Esclerosis Múltiple en Adultos.

Tabla 2. Preguntas clínicas PICO para Tratamiento de Esclerosis Múltiple

\begin{tabular}{llll}
\hline Población & Intervención & $\underline{\text { Comparador }}$ & $\underline{\text { O}}$ utcome \\
\hline & Interferón beta & & \\
& Acetato de glatirámero & & \\
& Teriflunomida & & Eficacia \\
& Fingolimod & & Tiempo de conversión a EMCD \\
& Natalizumab & & $\begin{array}{l}\text { Tasa anualizada de recaídas } \\
\text { Progresión de la enfermedad }\end{array}$ \\
& Alemtuzumab & Placebo & $\begin{array}{l}\text { Número de lesiones nuevas o en } \\
\text { crecimiento }\end{array}$ \\
Pacientes adultos $(>18$ años) & Rituximab & & \\
con esclerosis múltiple o & Prednisona & Interferón beta & Seguridad \\
síndrome clínico aislado & Metilprednisolona & (comparador activo) & Tasas de eventos adversos serios o \\
& Mitoxantrona & & casi fatales \\
& Azatioprina & & Discontinuación por eventos \\
adversos
\end{tabular}

Pregunta PICO:

En Población, ¿El uso de Intervención en comparación a Comparador reduce el Outcome?

Tabla 3. Criterios de McDonald 2010 para el diagnóstico de EM

Presentación clínica Datos adicionales necesarios para el diagnóstico de EM

$\geq 2$ o más ataques, evidencia clínica de $\geq 2$

lesiones o de 1 lesión con evidencia histórica Ninguno

razonable de un ataque previo

$\geq 2$ o más ataques, evidencia clínica de 1 lesión regiones (periventricular, yuxtacortical, infratentorial o médula espinal) o esperar un nuevo ataque que involucre una región distinta.

Diseminación en el tiempo: Presencia simultánea lesiones

1 ataque, evidencia clínica de $\geq 2$ lesiones asintomáticas captadoras y no captadoras de Gadolinio (Gd), o una nueva lesión en T2 y/o lesione(s) captadora(s) de Gd en RMN de seguimiento, o esperar por un segundo ataque

1 ataque, evidencia clínica objetiva de 1 lesión (síndrome clínico aislado)

Diseminación en tiempo y espacio

1 año de progresión de la enfermedad (retro o prospectivamente) + $\geq 2$ de los siguientes:

Progresión neurológica insidiosa sugerente de - Evidencia de diseminación en espacio (en el cerebro) basado en $\geq$ EM (EM primaria progresiva)

1 lesión en T2 en regiones características de EM

- Evidencia de diseminación en espacio en la médula espinal basado en $\geq 2$ lesiones en $\mathrm{T} 2$ a nivel espinal

- Bandas oligoclonales positivas* oíndice elevado de inmunoglobulina $\mathrm{G}^{* *}$ en líquido cefalorraquídeo (LCR)

\footnotetext{
*Bandas oligoclonales positivas: dos o más bandas que se encuentren en LCR y no en sangre ${ }^{32}$

**Índice elevado de inmunoglobulina G: > 0.7 o el valor definido por el laboratorio. ${ }^{33}$

Entre las otras pruebas que son de utilidad se incluyen el análisis del líquido cefalorraquídeo, la medición de potenciales evocados, la tomografía de coherencia óptica. ${ }^{34,35}$
} 
preguntas que conciernen al tratamiento (tabla 2). La población de interés fueron pacientes adultos con esclerosis múltiple en todas ellas y el subtipo clínico de la enfermedad fue especificado. Las intervenciones evaluadas fueron aquellas terapias modificadoras de la enfermedad aprobadas por aprobadas por DIGEMID hasta enero del 2017. Los comparadores en su mayoría se trataron de placebos, y para algunas preguntas, interferón beta fue usado como comparador activo. Los resultados (outcomes) que sirvieron para optar por la intervención o el comparador fueron planteados en base a las mediciones de eficacia y seguridad que se suelen realizar en ensayos clínicos. Estas preguntas fueron respondidas mediante meta-análisis hecho en el programa RevMan 5.3 empleando los ensayos clínicos disponibles hasta agosto del 2017. La calidad de estos además fue evaluada mediante la metodología $\operatorname{GRADE}(4,5,6)$.

El descriptor utilizado para la búsqueda de los estudios en Pubmed fue: multiple sclerosis, con los filtros "humans" OR "adult", "english" OR "spanish" OR "portuguese". Para las guías de prácticas clínicas, además, se usó el filtro "guideline" OR "practice guideline" OR "Dataset"; para las revisiones sistemáticas "systematic" OR "meta-analysis"; para los ensayos clínicos Clinical Trial OR Randomized Controlled Trial OR Pragmatic Clinical Trial OR Multicenter Study. Para las demás bases de datos se usaron los descriptores y filtros con igual significado. Las recomendaciones fueron elaboradas por el comité directivo y sometidas a escrutinio por el comité clínico mediante el uso del método Delphi modificado hasta alcanzar un consenso de al menos el $80 \%$ de la aprobación entre los miembros del comité clínico. La dirección de la recomendación estuvo dictada por la valoración entre el riesgo y beneficio de la intervención propuesta. La fuerza de la recomendación fue considerada como "fuerte" si la intervención propuesta probablemente no vaya a ser modificada con la adquisición de nueva evidencia. Y fue considerada como "débil" si es probable que la intervención se modifique con evidencia posterior. Las intervenciones fueron denominadas como de "buena práctica clínica" si eran consideradas beneficiosas y seguras, aun cuando haya escasa evidencia que respalde la misma. El manuscrito fue sometido a revisión externa por expertos internacionales en EM. En la elaboración de este documento se recogió la opinión de los pacientes con EM a través de las organizaciones ESMUP (Esclerosis Múltiple del Perú) y "Hechos con Amor", sobre los alcances y objetivos de la guía. La guía será difundida por medio electrónico a los jefes de servicio de neurología y estará disponible en libre acceso para el resto de profesionales médicos a través de la SPN. Se plantea su actualización cada dos a tres años por miembros de la SPN, manteniéndose las funciones del comité directivo.

\section{RESULTADOS}

\section{Preguntas clínicas

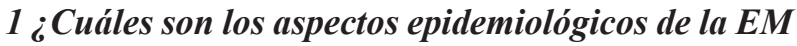 y sus factores de riesgo?}

La prevalencia global de la enfermedad es de 30 por cada 100000 habitantes (7). La distribución de los casos no es uniforme siendo mayor en países del

Tabla 4. Criterios para valorar la respuesta subóptima al tratamiento.

\section{Consenso del Foro Latinoamericano de Expertos en EM}

Si presenta uno o más de los anteriores

$>1$ recaída o 1 recaída severa

Recaída severa:

- recuperación incompleta a 6 meses.

- necesidad de hospitalización/esteroides.

Recaída - más de un sistema funcional afectado.

- compromiso severo motor, cerebeloso o de tronco encefálico.

- impacto significativo en la calidad de vida.

$\geq 2$ nuevas lesiones captadoras de $\mathrm{Gd}$

RMN $\quad \geq 2$ nuevas lesiones T2/año

$\geq 2$ lesiones en médula espinal

Cambio en EDSS de $\geq 2$ puntos sostenidos en 6 meses (aumento de 0.5 puntos si el EDSS es

Discapacidad mayor a 6.0)

Aumento de $20 \%$ en el tiempo de caminata de 25 pies (T25FW)* confirmado a 6 meses

*T25FW: el promedio del tiempo, en al menos dos ensayos, que demora un paciente en marchar, sin correr, una distancia de 25 pies de longitud con o sin dispositivos de asistencia, lo más rápido posible y de manera segura 
Guía de Práctica Clínica para el Diagnóstico y Tratamiento de Esclerosis Múltiple en Adultos.

Tabla 5. Recomendaciones del Grupo de Trabajo.

\section{Recomendaciones del Grupo de Trabajo}

\section{Fuerza de recomendación \\ Referencia}

\section{¿Cómo se realiza el diagnóstico de EM?}

Se deben emplear los criterios de McDonald 2010 para el diagnóstico de EM

Se sugiere que el tiempo de consulta previo al inicio de la TME sea de al menos treinta minutos

\section{¿Qué consideraciones se deben tener en cuenta previo al inicio de la TME?}

Se debe valorar el deseo gestacional previo al inicio de la TME en pacientes con EM

Se recomienda la medición de beta-hCG en sangre previo al inicio de la terapia modificadora de enfermedad en pacientes mujeres en edad reproductiva con EM

En mujeres en edad reproductiva se debe iniciar contracepción eficaz previo o concomitante al inicio de teriflunomida (durante y hasta que los niveles plasmáticos de teriflunomida se encuentren por debajo de $0.2 \mathrm{mg} / \mathrm{L}$ ), alemtuzumab (durante y 4 meses después), fingolimod (durante y 2 meses después) y natalizumab. En varones en edad reproductiva se debe iniciar contracepción eficaz previo o concomitante al inicio de teriflunomida (durante y hasta que los niveles plasmáticos de teriflunomida se encuentren por debajo de $0.2 \mathrm{mg} / \mathrm{L}$ )

Se sugiere discutir medidas de anticoncepción en pacientes en edad reproductiva con EM y seguir el plan de mitigación de riesgos ad hoc para cada droga

Se recomienda el tamizaje de enfermedad tuberculosa latente con las siguientes pruebas: radiografía de tórax, derivado puro proteico (PPD) o quantiferón

Se debe notificar a la autoridad de salud pertinente y referir para manejo conjunto especializado en caso de enfermedad tuberculosa en pacientes con EM
Fuerte

Buena práctica

clínica

Buena práctica

clínica

Fuerte

Buena práctica

clínica

Buena práctica

clínica

Fuerte

44

Buena práctica

clínica

En pacientes con SCA, ¿Cuál es el perfil de eficacia (retraso para la conversión a EMRR) y seguridad de las TME?

Se recomienda el uso de inmunomoduladores inyectables (interferón beta, acetato de glatirámero) o teriflunomida como TME en pacientes con SCA y lesiones compatibles con EM en la RMN

¿Cuál son los tratamientos disponibles para los brotes de la enfermedad?

Se recomienda el uso de pulsos de metilprednisolona y, en casos refractarios, plasmaféresis como terapia sintomática de la recaída en pacientes con EM

No se recomienda, necesariamente, el retiro gradual con corticoides orales luego de la terapia sintomática de la recaída

En pacientes con EMRR, ¿Cuál es perfil de eficacia y seguridad de las TME?

Se recomienda el uso de inmunomoduladores inyectables (interferón beta, acetato de glatirámero) o teriflunomida como TME, en pacientes con EMRR sin inicio agresivo Se recomienda el uso de fingolimod o anticuerpos monoclonales (natalizumab, alemtuzumab) como TME en pacientes con EMRR de inicio agresivo

Fuerte 56-61

Se recomienda el intercambio entre, interferón beta o acetato de glatirámero o teriflunomida en pacientes con EMRR y pobre tolerabilidad durante el tratamiento. (cambio lateral)

Se recomienda el uso de fingolimod o anticuerpos monoclonales (natalizumab, alemtuzumab) como TME en pacientes con EMRR con respuesta subóptima a teriflunomida o inmunomoduladores inyectables (interferón beta, acetato de glatirámero). (escalamiento)

Se sugiere el uso de rituximab como TME en pacientes con EMRR con respuesta subóptima a fingolimod y a anticuerpos monoclonales (natalizumab, alemtuzumab)

Fuerte

51

Buena práctica $\quad 55$ clínica

Fuerte

Fuerte $67-71$

Fuerte 
No se recomienda el uso de corticoides (prednisona, metilprednisolona) o inmunosupresores (azatioprina, micofenolato de mofetilo, ciclofosfamida, metrotexato) como TME en pacientes con EMRR

Se recomienda la medición de anticuerpos contra el virus John Cunningham para evaluar el riesgo de desarrollar leucoenfalopatía multifocal progresiva previo al inicio de tratamiento con natalizumab y cada 6 meses

Fuerte

Fuerte

73

74

\section{En pacientes con EM progresiva, ¿Cuál es perfil de eficacia y seguridad de las TME?}

No se recomienda el uso de inmunosupresores, inmunomoduladores (interferón beta, acetato de glatirámero), fingolimod o anticuerpos monoclonales (alemtuzumab, Fuerte natalizumab, rituximab) como TME en pacientes con EM progresiva sin actividad

Se sugiere el uso de $80 \mathrm{mg}$ de simvastatina al día como terapia neuroprotectora adyuvante en pacientes con EM progresiva

Débil

En pacientes con EMSP, se recomienda el uso de IFN- $\beta$ 1a y IFN- $\beta$ 1b, siempre y cuando haya presencia de actividad inflamatoria, definida como recaídas, episodios agudos o subagudos de nueva o incrementada disfunción neurológica seguidos por recuperación parcial o total, en ausencia de fiebre o infecciones y/o lesiones hiperintensas captadores de Gadolinio en T1 o lesiones hiperintensas nuevas o en crecimiento en $\mathrm{T} 2$

hemisferio norte e impresiona seguir una gradiente latitudinal decreciente conforme se acerca al Ecuador (8). En el Perú, la prevalencia de EM en Lima se estima en 7 casos por cada 100000 habitantes (9).

El subtipo clínico más frecuente es la forma remitente-recurrente (EMRR). Cerca de 15\% inician con un curso primariamente progresivo (EMPP), y $70 \%$ de los que inician con un curso EMRR, luego de 10-15 años de historia natural de la enfermedad desarrollarán el fenotipo progresivo (EMSP) (10).

Los factores de riesgo para el desarrollo de esclerosis múltiple que hasta la fecha han sido descritos son: infección por el virus Epstein-Barr en edad temprana (mononucleosis infecciosa), tabaquismo, obesidad, ingesta de sal elevada, bajo nivel sérico de vitamina D y polimorfismos genéticos (HLA-DR2, HLA-DRB1*15:01, HLA-A*02) (11-16).

\section{2 ¿Cuál es el cuadro clínico de la EM?}

Se caracteriza en la mayoría de los casos por presentar brotes o recaídas. Estos se manifiestan como la aparición de síntomas o signos de disfunción neurológica de por lo menos 24 horas de duración, o un deterioro significativo de síntomas preexistentes, previamente estabilizados durante al menos 30 días (17).

Existen también formas progresivas de la enfermedad, definida como la disfunción o discapacidad neurológica que incrementa constantemente, documentada de manera objetiva sin recuperación; fluctuaciones o fases de estabilidad pueden ocurrir. Adicionalmente, las formas progresivas pueden estar asociadas con actividad clínica o radiológica, particularmente en los estadios iniciales (17).

En el Perú, la edad de inicio de enfermedad fluctúa entre 28 y 32 años, siendo más común en mujeres. Las manifestaciones clínicas más frecuentes son motoras, sensitivas, neuritis óptica y síntomas troncales $(18,19)$.

\section{En pacientes con síndrome clínico aislado ¿es posible identificar factores de riesgo para progresión hacia esclerosis múltiple clínicamente definida?}

Entre las características sociodemográficas y clínicas, la edad menor a 30 años, el género femenino y el compromiso de varios sistemas funcionales, parecen ser los factores que aportan un mayor riesgo para la conversión a EMCD $(20,21)$. Por el contrario, el desarrollo de neuritis óptica como presentación inicial de la enfermedad tiene el menor riesgo de conversión a EMCD (22). La presencia de bandas oligoclonales en líquido cefalorraquídeo es un hallazgo que predice la conversión a EMCD de manera independiente del resto de factores pronósticos (23). Los individuos que cumplen 3 o 4 criterios de Barkhof en la RMN también tienen un elevado riesgo de conversión a EMCD (24). Estos criterios son los siguientes: 1) una lesión captadora de contraste $0 \geq 9$ lesiones en secuencia T2, 2) una lesión infratentorial, 3) una lesión yuxtacortical, 4) tres lesiones periventriculares. 
Guía de Práctica Clínica para el Diagnóstico y Tratamiento de Esclerosis Múltiple en Adultos.

4 En pacientes con esclerosis múltiple clínicamente definitiva ¿es posible identificar factores de riesgo para progresión de la enfermedad o para enfermedad agresiva?

Los factores sociodemográficos asociados con un riesgo elevado de progresión de la enfermedad y enfermedad agresiva son el inicio de síntomas desde los 40 años, el género masculino y la etnia afrolatinoamericana $(25,26)$.

El cuadro clínico al inicio de la enfermedad asociado a un peor pronóstico está constituido por síntomas motores, y síntomas derivados de afección del tronco encefálico y cerebelo. De igual forma están asociadas, la frecuencia de recaídas durante los primeros cinco años de la enfermedad, y el tiempo que transcurre entre estas $(25,26)$.

El número de lesiones y su localización, especialmente en región infratentorial, también permiten pronosticar un deterioro neurológico acelerado y sostenido de la enfermedad $(27,28)$.

\section{5 ¿Cómo se realiza el diagnóstico de EM?}

Los criterios de McDonald modificados el 2010 (tabla 3) unifican los hallazgos clínicos y radiológicos característicos de la enfermedad (29). Al tiempo de escribir este manuscrito, son los más aceptados a nivel mundial para el diagnóstico de esclerosis múltiple. Para el subtipo remitente recurrente se requiere evidencia clínica de al menos un ataque y lesiones en RMN con diseminación en el espacio y el tiempo. Para el subtipo progresivo se requiere, adicionalmente, deterioro neurológico sostenido por al menos un año. La sensibilidad y especificidad ha sido estimada en $84 \%$ y $80 \%$, respectivamente, en una población sudamericana (30).

Recientemente, se han propuesto nuevos criterios radiológicos por el grupo MAGNIMS con la finalidad de incrementar la especificidad del diagnóstico sin disminuir la sensibilidad en forma importante. Nuevos estudios serán necesarios para determinar con certeza la utilidad de esta propuesta (31).

\section{6 ¿Cuál es el rol de los exámenes auxiliares para el diagnóstico de EM?}

El rol que cumplen los exámenes auxiliares es como ayuda para el diagnóstico diferencial de esclerosis múltiple. Entre los exámenes auxiliares se encuentran las imágenes por RMN, los potenciales evocados somatosensoriales y el análisis de líquido cefalorraquídeo $(32,35)$.

\section{7 ¿Qué consideraciones se deben tener en cuenta previo al inicio de terapia modificadora de la enfermedad?}

Es fundamental que el médico tratante tome en consideración no sólo la eficacia, seguridad y tolerabilidad del tratamiento. El estilo de vida del paciente, sus comorbilidades y el costo del fármaco son factores que impactan poderosamente en la adherencia al tratamiento por lo que la elección del mismo debe ser una decisión compartida entre ambos. Es importante que cualquiera fuera esta decisión, el inicio del tratamiento sea rápido. Se sabe que el daño en las primeras etapas de la enfermedad tiene un fuerte impacto negativo en el pronóstico a largo plazo. Es bajo este concepto que en pacientes con EM de inicio agresivo; es decir, con rápido deterioro de la función neurológica y evidencia radiológica de actividad inflamatoria, la terapia modificadora de la enfermedad con drogas de alta eficacia, desde el inicio, está justificado (36).

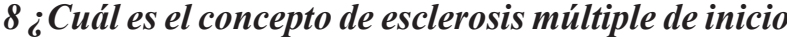 agresivo y su importancia?}

La esclerosis múltiple de inicio agresivo se caracteriza por el deterioro rápido de la función neurológica con evidencia de actividad inflamatoria descontrolada en forma de brotes con acúmulo de lesiones en la RMN. Es importante identificar este subgrupo de pacientes para que el inicio del tratamiento sea con fármacos de alta eficacia $(28,37)$.

\section{9 ¿Cuál es la conducta terapéutica a seguir en mujeres con deseo gestacional, gestantes y puérperas con esclerosis múltiple?}

En mujeres en edad reproductiva es prudente valorar el deseo gestacional y solicitar el descarte de embarazo previo al inicio de cualquier fármaco (39). En mujeres en edad reproductiva se debe iniciar contracepción eficaz previo o concomitante al inicio de teriflunomida (durante y hasta que los niveles plasmáticos de teriflunomida se encuentren por debajo de $0,2 \mathrm{mg} / \mathrm{L}$ ), alemtuzumab (durante y 4 meses después), fingolimod (durante y 2 meses después) y natalizumab. En varones en edad reproductiva se debe iniciar contracepción eficaz previo o concomitante al inicio de teriflunomida (durante y hasta que los niveles 
plasmáticos de teriflunomida se encuentren por debajo de $0,2 \mathrm{mg} / \mathrm{L})(40-43)$.

\section{En los pacientes con EM en terapia modificadora de la enfermedad ¿cuál es el riesgo de desarrollar enfermedad tuberculosa primaria o reactivada?}

Dada la alta prevalencia de tuberculosis en el Perú, la terapia modificadora de la enfermedad con inmunosupresores e inmunomoduladores podría elevar el riesgo de reactivación para enfermedad tuberculosa latente, y posiblemente, el riesgo de infección primaria. El tamizaje con radiografía de tórax, derivado puro proteico (PPD) o quantiferon es imprescindible previo al inicio del tratamiento y potencialmente durante el seguimiento (44).

El tratamiento con corticoides y alemtuzumab tienen un alto riesgo de reactivación de enfermedad tuberculosa $(45,46)$. Se ha descrito un riesgo potencial con el uso de fingolimod y teriflunomida debido al mecanismo de acción de estos fármacos. Por el contrario, no se ha demostrado riesgo de reactivación con el uso de interferón beta, acetato de glatirámero, y mitoxantrona.

11 ¿Cuáles son los criterios de respuesta subóptima y la importancia de la adherencia a la terapia modificadora de la enfermedad?

La respuesta subóptima al tratamiento es valorada mediante el Consenso del Foro Latinoamericano de Expertos en Esclerosis Múltiple detallados en la tabla 4 (36).

12 ¿Cuáles son las condiciones de escalamiento o cambio lateral en la terapia modificadora de la enfermedad en pacientes con esclerosis múltiple?

Las condiciones que justifican el escalamiento hacia fármacos de alta eficacia son la respuesta subóptima. El cambio lateral entre fármacos de similar eficacia con mejor tolerabilidad se argumenta en la pobre tolerabilidad al tratamiento actual. Las preferencias del paciente deben ser evaluadas previo al inicio y durante la terapia modificadora de la enfermedad $(47,48)$.

\section{3 ¿Cuál es el marco de la prescripción de los medicamentos biosimilares?}

La directiva queda regulada mediante el Decreto Supremo No 013-2016-SA. El mismo que se encuentra disponible en http://busquedas.elperuano.com.pe/ normaslegales/aprueban-reglamento-que-regula-la- presentacion-y-contenido-d-decreto-supremo-n-0132016-sa-1350762-1/

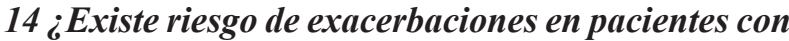 esclerosis múltiple luego de la vacunación?}

En pacientes con esclerosis múltiple que reciben terapia modificadora de la enfermedad no es recomendable la vacunación con microorganismos vivos atenuados. Si la vacunación está indicada debe seguir el plan de manejo de riesgo descrita para cada terapia modificadora de la enfermedad. Las vacunas contra los siguientes patógenos: bacilo de calmetteguérin (BCG), hepatitis viral B (HBV), sarampiónrubéola-parotiditis (SPR), varicela zoster (VZV), papiloma virus humano (HPV), tétanos, influenza y rabia no han demostrado un incremento en el riesgo de exacerbaciones. La evidencia es insuficiente para garantizar la seguridad del resto de vacunas $(49,50)$.

15 ¿Cuáles son los tratamientos disponibles para el brote de la enfermedad y cuál es el más adecuado?

La exacerbación o recaída en el curso de la enfermedad suele responder a dosis de 500-1000 mg de metilprednisolona EV por tres a cinco días. En pacientes en los que no se encuentra respuesta, la plasmaféresis es una alternativa, no así el uso de dexametasona o inmunoglobulina. El retiro gradual con corticoides orales luego de los pulsos de metilprednisolona tampoco ha demostrado, necesariamente, algún beneficio adicional (51-55).

16 En pacientes con síndrome clínico aislado ¿cuál es el perfil de eficacia y seguridad de las terapias modificadoras de la enfermedad?

Los ensayos clínicos TOPIC, PreCISe, BENEFIT, CHAMPS, ETOMS y REFLEX, son algunos de los que han demostrado que el uso de TME extienden el tiempo de conversión a EM en pacientes con SCA, para drogas como teriflunomida, acetato de glatirámero e interferón beta, respectivamente (56-61).

\section{7 ¿Cuál es el perfil de eficacia y seguridad de las TME para el tratamiento de EMRR?}

Las drogas que han demostrado modificar la historia natural de la enfermedad en pacientes con EMRR son interferón beta, acetato de glatirámero, teriflunomida, fingolimod, natalizumab y alemtuzumab. Los ensayos clínicos MSCRG, PRISMS, MSSG, CMSSG, TEMSO, TOWER, FREEDOMS, AFFIRM y CARE 
Guía de Práctica Clínica para el Diagnóstico y Tratamiento de Esclerosis Múltiple en Adultos.

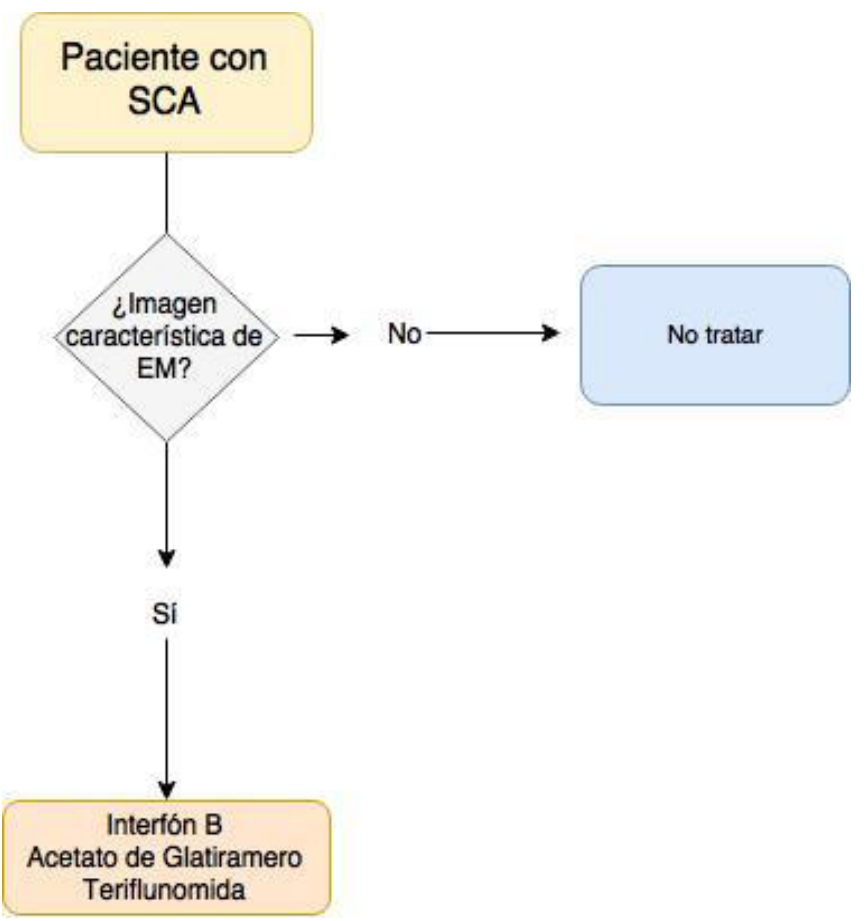

Figura 1. Algoritmo de manejo para pacientes con Síndrome Clínico Aislado

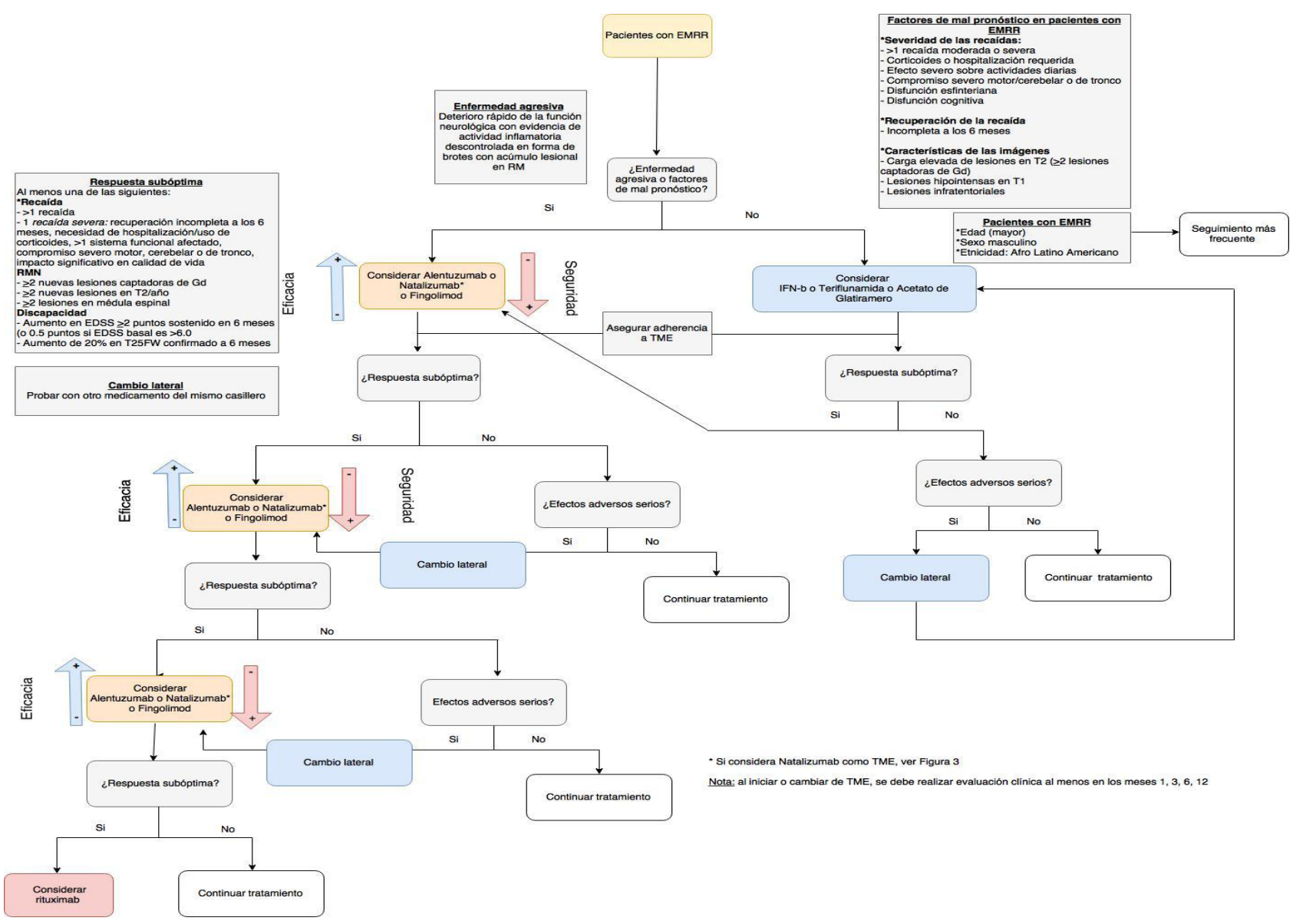

Figura 2. Algoritmo de manejo para pacientes con Esclerosis Múltiple Recurrente Remitente 


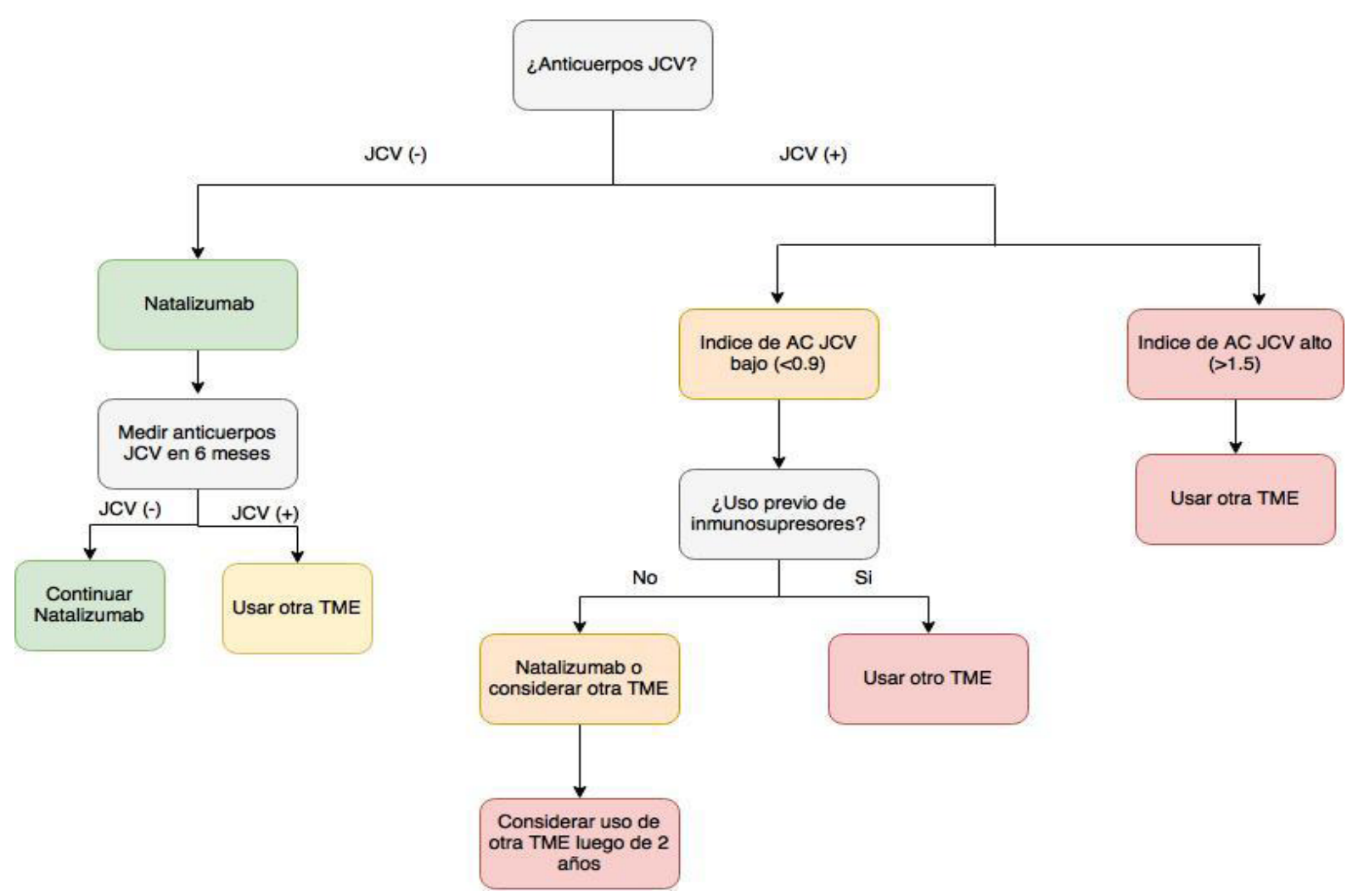

Figura 3. Algoritmo para el uso de Natalizumab

MS, respectivamente, han reportado reducir la tasa anual de recaídas y la acumulación sostenida de la discapacidad en comparación a placebo, y algunos de ellos frente a comparador activo (62-71).

\section{8 ¿Cuál es el perfil de eficacia y seguridad de las TME para el tratamiento de EMPP y EMSP?}

No se ha podido replicar el resultado obtenido con el uso de inmunomoduladores o inmunosupresores que ha sido visto en pacientes con EMRR. Los ensayos clínicos que evaluaron la eficacia de las drogas más recientes para el curso progresivo de la enfermedad, como los que emplearon fingolimod, natalizumab y rituximab (INFORMS, ASCEND, OLYMPUS; respectivamente) no demostraron beneficio (7279). No obstante, algunos estudios han reportado algún beneficio de fármacos como ocrelizumab (ORATORIO) (80), biotina en una presentación no disponible en el Perú o simvastatina como agentes neuroprotectores $(81,82)$.

El resumen de las recomendaciones elaboradas por el Grupo de Trabajo se encuentra en la tabla 5 y en los algoritmos detallados en las figuras 1, 2 y 3 .

\section{Aplicabilidad y Limitaciones}

Se describen algoritmos de tratamiento para favorecer la aplicabilidad de las recomendaciones. Entre las limitaciones, la siguiente guía no ha considerado la evidencia que concierne a población pediátrica ni el tratamiento de los síntomas derivados de las complicaciones de la EM. Por otro lado, la ausencia de estudios de costo efectividad locales limita el análisis sobre los recursos que deberían ser aplicados para implementar las recomendaciones, sin embargo, las referencias internacionales sustentan el beneficio de las terapias modificadoras de la enfermedad para los pacientes y los sistemas de salud (84-87).

\section{Correspondencia:}

Darwin R. Vizcarra.

Hypnos Instituto del Sueño-Clínica San Felipe.

Correo electrónico: dvizcarra@yahoo.com

\section{Financiamiento:}

Esta no contó con financiamiento privado o estatal para su elaboración. Los comités directivo, clínico y panel de revisores externos no recibieron pago alguno por su actividad, solo se tuvo un fondo ad hoc de la SPN para cubrir los gastos del comité investigador. 
Guía de Práctica Clínica para el Diagnóstico y Tratamiento de Esclerosis Múltiple en Adultos.

\section{Conflicto de intereses:}

Darwin Roger Vizcarra Escobar ejerció como advisory board en Genzyme y Roche. Ha sido speaker de Stendhal, y participa como investigador principal en un ensayo clínico multicéntrico financiado por Novartis. Ha recibido apoyo para asistir a congresos por Roche y Merck. César Caparó Zamalloa es Speaker para los siguientes laboratorios: Merck Serono, Bayer, Novartis, Stendhal, Genzyme. Ha recibido apoyo para participación en congresos de los siguientes laboratorios: Merck Serono, Novartis, Genzyme y Stendhal. Carlos César Castañeda Barba ha participado en ensayos clínicos como investigador principal o subinvestigador en estudios auspiciados por Merck, Novartis, Roche, Biogen. Ha prestado servicios como speaker o advisory board para compañías como Stendhal, Genzyme, Merck, Biogen. He recibido auspicios para asistir a congresos o cursos internacionales o nacionales de empresas como Biogen, Genzyme, Grunenthal, Merck, Novartis, Stendhal. Sheila Castro Suárez ha recibido apoyo para asistir a congresos y cursos por Genzyme, Novartis, Stendhal, Merck y Galenicum. Ana Graciela Cruz Cruz participa como investigadora principal y sub-investigadora en ensayos clínicos multicéntricos financiados por Novartis y Roche. Ha participado como investigadora en estudios patrocinados por el NIH y otros auspiciados directamente o a través de CRO por Abbot, Biogen y Merck. Ha prestado servicios como advisory board en Genzyme y Teva. Ha sido speaker de Bayer, Genzyme, Novartis, Roche, Stendhal y Teva. Recibió auspicio para asistir a cursos y congresos de Abbott, Bayer, Genzyme Novartis, Stendhal, Merck Serono, Medco y Roche. Jorge Martín Gavidia Chucán es Advisory Board por Genzyme, Recibió auspicio para asistir a congresos internacionales de neurología por Genzyme, Merck Serono, Novartis, Boehringer Ingelheim. Fue Speaker para Sanofi, Merck, Pfizer y Genzyme. Participó en ensayos clínicos financiados por: Biogen, Roche, Pfizer, Merck Sharp\&Dome, Novartis, AstraZeneca, el NIH y CROs. Oscar González Gamarra ha sido speaker para Genzyme y Novartis. Ha recibido apoyo de Novartis y Genzyme para asistir a Congresos. Marco Huertas Navarro Ha recibido apoyo financiero para congreso por Stendhal Peru. María Meza Vega Ha asistido a dos cursos de Esclerosis múltiple invitada por la industria farmacéutica (GENZYME) en el 2015. Asistió a un curso sobre Esclerosis patrocinados por la industria farmacéutica. Ha asistido a dos cursos sobre Esclerosis Múltiple patrocinado por Colombia (STENDHAL) y Brazil104 (MERCK).
Asistió al ECTRIMS realizado en Barcelona-España, patrocinada por la industria farmacéutica (BAYER). Nicanor Mori Quispe ejerció como Médico de Enlace Científico para Esclerosis Múltiple en Laboratorios Novartis Biosciences Peru S.A. Ha dictado charlas para Laboratorios Abbott, Axon Pharma y Roemmers. Recibió honorarios como sub-investigador por Laboratorios AztraZeneca. Ha recibido apoyo para asistencia a congresos por GlaxoSmithKline, Teva, Siegfried y Grunenthal y Galenicum. Edgar Rojas Huerto, ha recibido honorarios como conferencista sobre Esclerosis múltiple por Bayer, como clinical advisor por Genzyme. Ha recibido financiamiento para congresos (LACTRIMS, ECTRIMS, ACTRIMS) por parte de BAYER S. A., STHENDAL S. A. TEVA S. A y TECNOFARMA S.A. y trabaja como sub-investigador clínico en estudios financiados por Novartis. Victor Montalván Ayala, Alda Rivara Castro, Elsa Rosa Neira Sánchez, Fiorella Adrianzén Álvarez, Sebastian A. Serván Lozano, Joaquín A. Vizcarra Pasapera, Brian J. Villafuerte Trisolini, Kevin Duque Yupanqui y Rodrigo Zamudio Herrera no tienen conflicto de intereses que declarar. Oscar Fernández ha recibido honorarios como consultor en juntas de asesoramiento y como moderador $\mathrm{u}$ orador en congresos, también ha participado en ensayos clínicos y otros proyectos de investigación promocionados por Actelión, Allergan, Almirall, Biogen, Bayer-Schering, Genzyme, Merck-Serono, Novartis, Teva y Roche. Víctor Rivera es miembro del Speakers Bureau: EMD Serono, Sanofi-Genzyme. Medical Writing Honorarium: Biogen. Asesor de Advance-Medical. Auspicios del Consortium of MS Centers. Adriana Carrá es miembro asociado a Biosidus, Gador, Gemabiotech, Genzyme Argentina/LATAM, Merck Argentina/LATAM, MS Synthon-Bago, Roche, Teva Global, TEVA Argetina, Tuteur. También ha recibido honorarios por su labor en ponencias para Bayern, Biogen, Biosidus, Novartis.

\section{REFERENCIAS BIBLIOGRAFICAS}

1. Ministerio de Salud. Norma Técnica de Salud para la elaboración y uso de guías de práctica clínica. Lima: Ministerio de Salud; 2015.

2. Brouwers M, Kho ME, Browman GP, et al. On behalf of the AGREE Next Steps Consortium. AGREE II: Advancing guideline development, reporting and evaluation in healthcare. Can Med Assoc J. 2010; 182:E839-842

3. Agencia de Información Evaluación y Calidad en Salud de Cataluña. Guía de práctica clínica sobre la atención a las personas con esclerosis múltiple. 
Barcelona: Agencie d'Informació A i Q, en Salut Servei Catala de la Salut. Departament de Salut. Generalitat de Catanlunya; 2012.

4. Higgins JPT, Green S (editors). Cochrane Handbook for Systematic Reviews of Interventions Version 5.1.0 Londres: The Cochrane Collaboration; 2011.

5. Schunemann H, Brozek J, Guyatt G, et al. GRADE Handbook. New Tork: GRADE Working Group; 2013.

6. Guyatt G, Oxman AD, Akl EA, et al. GRADE Guidelines. J Clin Epidemiol. 2011;64(4):383-94. doi: 10.1016/j.jclinepi.2010.04.026

7. Multiple Sclerosis International Federation. Atlas of MS 2013: Mapping Multiple Sclerosis around the World. New York: Mult Scler Int Fed; 2013.p. $1-28$

8. Simpson S, Bizzard L, Otahal P, van der Mei, Taylor B. Latitude is significantly associated with the prevalence of multiple sclerosis: a metaanalysis. J Neurol Neur Psychiatry. 2011; 82(10):1132-41.

9. Vizcarra D, Kawano J, Castañeda C, Chereque A, Tipismana M, Bernabe A, et al. Prevalencia de Esclerosis Múltiple en Lima-Perú. Rev Médica Hered 2009. 20(3): 146-50.

10. Miller DH, Leary SM. Primary-progressive multiple sclerosis. Lancet Neurol. 2007; 6:903-12.

11. Belbasis L, Bellou V, Evangelou E, Ioannidis JPA, Tzoulaki I. Environmental risk factors and multiple sclerosis: An umbrella review of systematic reviews and meta-analysis. Lancet Neurol. 2015; 14(3):263-73.

12. Handel AE, Williamson AJ, Disanto G, Dobson R, Giovannoni G, Ramagolapan SV. Smoking and multiple sclerosis: An updated meta-analysis. PLoS One. 2011; 6(1):2-7.

13. Langer-Gould A, Brara SM, Beaber BE, Koebnick C. Childhood obesity and risk of pediatric multiple sclerosis and clinically isolated syndrome. Neurology. 2013; 80(6):548-52

14. McDonald J, Graves J, Waldman A. A case-control study of dietary salt intake in pediatric-onset multiple sclerosis. Mult Scler Relat Disord. 2016; 6:87-92.

15. Duan S, Lv Z, Fan X. Vitamin D status and the risk of multiple sclerosis: a systematic review and meta-analysis. Neurosci Lett. 2014; 570:108-13.

16. Godde R, Rohde K, Becker C. Association of the HLA region with multiple sclerosis as confirmed by a genome screen using $>10,000$ SNPs on DNA chips. J Mol Med. 2005; 83(6):486-94.

17. Lublin FD, Reingold SC, Cohen JA. Defining the clinical course of multiple sclerosis: the 2013 revisions. Neurology. 2014; 83:278-86.

18. Vizcarra D, Cava L, Tipismana M. Esclerosis múltiple en el Perú. Descripción clinicoepidemiológica de una serie de pacientes. Rev Neurol. 2005; 41(10):591-5.

19. Rojas E, Rentería J, Gonzales O. Epidemiological characteristics of Multiple Sclerosis in Perú. Montreal Canadá: Poster P577 Actrims, Ectrims, Lactrims; 2008.

20. Miller DH, Chard DT, Ciccarelli O. Clinically isolated syndromes. Lancet Neurol. 2012; 11(2):157-69.

21. D'Alessandro R, Vignatelli L, Lugaresi A. Risk of multiple sclerosis following clinically isolated syndrome: A 4-year prospective study. J Neurol. 2013; 260(6):1583-93.

22. Tintore M, Rovira A, Rio J. Is optic neuritis more benign tan other first attacks in multiple sclerosis? Ann Neurol. 2005; 57(2):210-5.

23. Dobson R, Ramagopalan S, Davis A. Cerebrospinal fluid oligoclonal bands in multiple sclerosis and clinically isolated syndromes: a meta-analysis of prevalence, prognosis and effect of latitude. $\mathrm{J}$ Neurol Neurosurg Psychiatry. 2013; 84(8):909-14.

24. Barkhof F, Filippi M, Miller DH. Comparison of MRI criteria at first presentation to predict conversion to clinically definitive multiple sclerosis. Brain. 1997; 120(11):2059-69.

25. Vasconcelos CCF, Aurencao JCK, Thuler LCS. Prognostic factors associated with long-term disability and secondary progression in patients with multiple sclerosis. Mult Scler Relat Disord. 2016; 8:27-34.

26. Damasceno A, von Glehn F, Brandao CO. Prognostic indicators for long-term disability in multiple sclerosis patients. J Neurol Sci. 2013; 324(1-2):29-33.

27. Minneboo A, Barkhof F, Polman CH. Infratentorial Lesions Predict Long-term Disability in Patients with Initial Findings Suggestive of Multiple Sclerosis. Arch Neurol. 2004; 61:217-21.

28. Freedman MS, Rush CA. Severe, Highly Active, or Aggressive Multiple Sclerosis. Contin Lifelong Learn Neurol. 2016; 22(3):761-84.

29. Polman CH, Reingold SC, Banwell B. Diagnostic criteria for multiple sclerosis: 2010 revisions to the McDonald criteria. Ann Neurol. 2011; 69:292.

30. Patrucco L, Rojas J, Miguez J. Application of the McDonald 2010 criteria for the diagnosis of multiple sclerosis in an Argentinian cohort of patients with clinically isolated síndromes. Mult Scler J. 2013; 19(10):1297-301. 
Guía de Práctica Clínica para el Diagnóstico y Tratamiento de Esclerosis Múltiple en Adultos.

31. Fillipi M, Rocca MA, Ciccarelli O. MRI criteria for the diagnosis of multiple sclerosis: MAGNIMS consensus guidelines. Lancet Neurol. 2016; 4422(15):1-12.

32. Dobbson R, Ramagopalan S, Davis A, Giovannoni G. Cerebrospinal fluid oligoclonal bands in multiple sclerosis and clinically isolated syndromes: a meta-analysis of prevalence, prognosis and effect of latitude. J Neurol Neurosurg Psychiatry. 2013; 84:909.

33. Rammohan KW. Cerebrospinal fluid in multiple sclerosis. Ann Indian Neurol. 2009; 12(4): 246253.

34. Pelavo R, Montalban X, Minoves T. Do multimodal evoked potentials add information to MRI in clinically isolated syndromes? Mult Scler. 2010; 16(1): 55-61.

35. Giesser BS. Diagnosis of multiple sclerosis: Neurol Clin 2011; 29:281.

36. Correale J, Abad P, Alvarenga R. Management of relapsing-remitting multiple sclerosis in Latin America: Practical recommendations for treatment optimization. J Neurol Sci. 2014; 339(1-2): 196206.

37. García A, Ara J, Fernández O. Consenso para el tratamiento de la esclerosis múltiple 2016. Sociedad Española de Neurología. Neurología. 2017; 32(2):113-9.

38. Buraga I, Popovici RE. Multiple sclerosis and pregnancy: current considerations. The Scientific World Journal. 2014; 2014: 160-166.

39. Fragoso YD, Boggild M, Maclas-Islas MA. The effect of long-term exposure to disease-modifying drugs during pregnancy in multiple sclerosis. Clin Neurol Neurosurg. 2013; 115(2):154-9.

40. SANOFI. Aubagio (Teriflunomide) product information. Paris: SANOFI; 2012

41. Sanofi Belgium. Lemtrada (Alemtuzumab) product information. Belgica: Sanofi Belgium; 2014

42. Sanofi-Aventis. Gilenya (Fingolimod) capsules product information. Paris: Sanofi-Aventis; 2016.

43. Friend S, Richman S, Bloomgren G. Evaluation of pregnancy outcomes from the Tysabri ${ }^{\circledR}$ pregnancy exposure registry: a global, observational, follow-up study. BMC Neurol. 2016; 16(1): 150 .

44. Fragoso YD, Adoni T, Anacleto A. How do we manage and treat a patient with multiple sclerosis at risk of tuberculosis? Expert Rev Neurother. 2014 14(11), 1251-1260.

45. Jick SS, Lieberman ES, Rahman MU. Glucocorticoid use, other associated factors, and the risk of tuberculosis. Arthritis Care Res
(Hoboken). 2006; 55(1):19-26.

46. Au WY, Leung AY, Tse EW. High incidence of tuberculosis after alemtuzumab treatment in Hong Kong Chinese patients. Leuk Res. 2008; 32(4):54751.

47. Freedman MS, Selchen D, Arnold DL. Treatment optimization in MS: Canadian MS Working Group updated recommendations. Can J Neurol Sci. 2013; 40(3):307-23.

48. Ingwersen J, Aktas O, Hartung HP. Advances in and Algorithms for the Treatment of RelapsingRemitting Multiple Sclerosis. Neurotherapeutics. 2016; 13(1):47-57.

49. Rutschmann OT, McCrory DC, Matchar DB. Immunization Panel of the Multiple Sclerosis Council for Clinical Practice Guidelines. Immunization and MS: a summary of published evidence and recommendations. Neurology. 2002; 59(12):1837-43.

50. Williamson EML, Chahin S, Berger JR. Vaccines in Multiple Sclerosis. Curr Neurol Neurosci Rep. 2016; 16(4):36.

51. Miller DM, Weinstock-Guttman B, Bethoux F. A meta-analysis of methylprednisolone in recovery from multiple sclerosis exacerbations. Mult Scler. 2000; 6(4):267-73.

52. Filippini G, Brusaferri F, Sibley WA. Corticosteroids or ACTH for acute exacerbations in multiple sclerosis. Cochrane Database Syst Rev. 2000; 4:1331.

53. De Keyser J, Zwanikken CM, Zorgdrager A. Treatment of acute relapses in multiple sclerosis at home with oral dexamethasone: a pilot study. J Clin Neurosci. 1999; 6(5):382-4.

54. Weinshenker BG, O'Brien PC, Petterson TM. A randomized trial of plasma exchange in acute central nervous system inflammatory demyelinating disease. Ann Neurol. 1999; 46(6):878-86.

55. Perumal JS, Caon C, Hreha S, Zabad R, Tselis A, Lisak R, et al. Oral prednisone taper following intravenous steroids fails to improve disability or recovery from relapses in multiple sclerosis. Eur J Neurol. 2008;15(7):677-80.

56. Miller AE, Wolinsky JS, Kappos L. Oral teriflunomide for patients with a first clinical episode suggestive of multiple sclerosis (TOPIC): a randomized, double-blind, placebo-controlled, phase 3 trial. Lancet Neurol. 2014; 13: 977-86.

57. Comi G, Martinelli V, Rodegher M. Effect of glatiramer acetate on conversion to clinically definitive multiple sclerosis in patients with clinically isolated syndrome (PreCISe study): a randomized, double-blind, placebo-controlled 
trial. Lancet. 2009; 374: 1503-11.

58. Kappos L, Polman CH, Freedman MS. Treatment with interferon beta- $1 \mathrm{~b}$ delays conversion to clinically definitive and McDonald MS in patients with clinically isolated syndromes. Neurology. 2006; 67:1242-49.

59. Jacobs LD, Beck RW, Simon JH. Intramuscular interferon beta-1a therapy initiated during a first demyelinating event in multiple sclerosis. CHAMPS Study Group. N Engl J Med. 2000; 343; 2219-26.

60. Comi G, Filippi M, Barkhof F. Effect of early interferon treatment on conversion to definitive multiple sclerosis: a randomized study. Lancet. 2001; 357: 1576-82.

61. Comi G, De Stefano N, Freedman MS. Comparison of two dosing frequencies of subcutaneous interferon beta-1a in patients with a first clinical demyelinating event suggestive of multiple sclerosis (REFLEX): a phase 3 randomized controlled trial. Lancet Neurol. 2012; 11: 33-41.

62. Jacobs LD, Cookfair DL, Rudick RA. Intramuscular interferon beta-1a for disease progression in relapsing multiple sclerosis. Ann Neurol. 1996; 39: 285-94.

63. Prevention of Relapses and Disability by Interferon beta-1a Subcutaneously in Multiple Sclerosis Study Group. Randomized double-blind placebocontrolled study of interferon beta-1a in relapsing/ remitting multiple sclerosis. Lancet. 1998; 352: 1498-504.

64. Duquette P, Girard M, Despault L. Interferon beta$1 \mathrm{~b}$ is effective in relapsing-remitting multiplesclerosis - clinical-results of a multicenter, randomized, double-blind, placebo-controlled trial. Neurology. 1993; 43: 655-61.

65. O'Connor P, Wolinsky JS, Confavreux C. Randomized trial of oral teriflunomide for relapsing multiple sclerosis. N Engl J Med. 2011; 265: 1293303.

66. Confavreux C, O'Connor P, Comi G. Oral teriflunomide for patients with relapsing multiple sclerosis (TOWER): a randomized, double-blind, placebo-controlled, phase 3 trial. Lancet Neurol. 2014; 13: 247-56.

67. Kappos L, Radue EW, O'Connor PW. A placebocontrolled trial of oral fingolimod in relapsing multiple sclerosis. N Engl J Med. 2010; 362: 387401.

68. Calabresi PA, Radue EW, Goodin D. Safety and efficacy of fingolimod in patients with relapsingremitting multiple sclerosis (FREEDOMS II): a double-blind, randomized, placebo-controlled, phase 3 trial. Lancet Neurol. 2014; 13: 545-56.

69. Polman CH, O'Connor PW, Havrdova E. A randomized, placebo-controlled trial of natalizumab for relapsing multiple sclerosis. $\mathrm{N}$ Engl J Med. 2006; 354: 899-910.

70. Cohen JA, Coles AJ, Arnold DL. Alemtuzumab versus interferón beta 1a as first-line treatment for patients with relapsing-remitting multiple sclerosis: a randomized controlled phase 3 trial. Lancet. 2012; 380: 1819-28.

71.Coles AJ, Twyman CL, Arnold DL. Alemtuzumab for patients with relapsing multiple sclerosis after disease-modifying therapy: a randomized controlled phase 3 trial. Lancet. 2012; 380: 18291839.

72. Hauser SL, Waubant E, Arnold DL. B-cell depletion with rituximab in relapsing-remitting multiple sclerosis. N Engl J Med. 2008; 358: 67688.

73. Filippini G, DelGiovane C, Vacchi L. Inmunomodulators and inmunosuppresants for multiple sclerosis: a network meta-analysis (Review). Cochrane Databasa Syst Rev. 2013; 6(6): 8933.

74. SorensenPS, BertolottoA,EdanG.Riskstratification for progressive multifocal leukoencephalopathy in patients treated with natalizumab. Mult Scler. 2012. 18:143.

75.Lublin F, Miller DH, Freedman MS. Oral fingolimod in primary progressive multiple sclerosis (INFORMS): a phase 3, randomized, double-blind, placebo-controlled trial. Lancet. 2016; 387: 1075-1084.

76. Steiner D, Arnold D, Freedman M. Natalizumab versus placebo in patients with secondary progressive multiple sclerosis (SPMS): results from ASCEND, a multicenter, double-blind, placebo-controlled, randomized phase 3 clinical trial. Vancouver, Canada: Annual Meeting of the American Academy of Neurology; April 15-21, 2016

77. Hawker K, O'Connor P, Freedman MS. Rituximab in patients with primary progressive multiple sclerosis. Results of a randomized double-blind, placebo-controlled multicenter trial. Ann Neurol. 2009; 66: 460-71.

78. La Mantia L, Vacchi L, Di Pietrantonj C. Interferon beta for secondary progressive multiple sclerosis. Cochrane Database Syst Rev 2012; 1: 5181.

79. Wolinsky JS, Narayana PA, O'Connor P. Glatiramer acetate in primary progressive multiple sclerosis: results of a multinational, multicenter, doubleblind, placebo-controlled trial. Ann Neurol. 2007; 
61: $14-24$

80. Montalban X, Hemmer B, Rammohan K. Efficacy and safety of ocrelizumab in primary progressive multiple sclerosis - results of the placebocontrolled, double-blind, phase III ORATORIO study (Abstract 228). Mult Scler J. 2015; 23: 78081 .

81. Sedel F, Papeix C, Bellanger A. High doses of biotin in chronic progressive multiple sclerosis: a pilot study. Mult Scler Relat Disord. 2015; 4(2): 139 .

82. Chataway J, Schuerer N, Alsanousi A. Effect of high-dose simvastatin on brain atrophy and disability in secondary progressive multiple sclerosis (MS-STAT): a randomized placebo-controlled, phase 2 trial. Lancet. 2014; 383(9936):2213.

83. Vizcarra-Escobar D, Mendiola-Yamasato A, Anculle-Arauco V. Treatment issues in Multiple Sclerosis in Latin America. Neuroepidemiology. 2015; 44: 66-68.
84. National Institute for Health and Care Excellence. Alemtuzumab for treating relapsing-remitting multiple sclerosis: Technology appraisal guidance [TA312]. Washington DC: National Institute for Health and Care Excellence NICE; 28 May 2014.

85. National Institute for Health and Care Excellence. Teriflunomide for treating relapsing-remitting multiple sclerosis: Technology appraisal guidance [TA303]. Washington DC: National Institute for Health and Care Excellence;22 January 2014.

86. National Institute for Health and Care Excellence. Fingolimod for the treatment of highly active relapsing-remitting multiple sclerosis. Technology appraisal guidance [TA254]. Washington DC: National Institute for Health and Care Excellence;25 April 2012.

87. National Institute for Health and Care Excellence. Natalizumab for the treatment of adults with highly active relapsing-remitting multiple sclerosis. Technology appraisal guidance [TA127]. Washington DC: National Institute for Health and Care Excellence; Published date: 22 August 2007. 